\title{
Research on O2O Supply Chain Decision Under the Condition of Trade-In
}

\begin{abstract}
Xuanzhi Wang
Business School of Sichuan University, Chengdu, Sichuan 610064

Corresponding author. Email: wangxuanzhi666@163.com

ABSTRACT

With the development of the times, today's society pays more and more attention to climate change, and carbon emission management is becoming more and more important. o2o mode has risen with the development of e-commerce in recent years, which has become an opportunity for many manufacturing enterprises to transform. This paper takes an o2o supply chain which is dominated by retailers and followed by manufacturers as the research object, and explores the pricing decisions of retailers and manufacturers in the supply chain of o2o under the condition that remanufacturing is based on the old for new and carbon tax rules. Through the model analysis, the optimal production and pricing strategies under different conditions are obtained with the change of production cost and carbon tax rate.
\end{abstract}

Keywords: Trade-in, Online to Offline supply chain, remanufacturing, carbon tax

\section{以旧换新条件下 020 供应链决策探究}

\author{
王炫植
}

四川大学 商学院, 四川成都 610064

通讯作者电子邮箱: wangxuanzhi666@163.com

摘 要

随着时代的发展, 当今社会愈发关注与重视气候变化, 碳排放管理愈发重要, $\mathrm{O} 2 \mathrm{O}$ 模式近年来随着电 商的发展而兴起, 成为许多制造企业转型的契机。本文以一条由零售商主导, 制造商跟随组成的 $\mathrm{O} 2 \mathrm{O}$ 供应链为研究对象, 探究再制造以旧换新、碳税规则的条件下 $\mathrm{O} 2 \mathrm{O}$ 供应链零售商与制造商的定价决策。 通过建立模型分析，得出随着生产成本和碳税率变化，不同条件下的最优生产与定价策略。

关键词: 以旧换新； $O 2 O$ 供应链；再制造；碳税

\section{1.引言}

$\mathrm{O} 2 \mathrm{O}$ 模式是近年来崛起的一种线上到线下 (Online to Offline）的商务模式, 用来解决纯线上渠道带来的购 物体验差, 服务质量难以提高的问题, 是一种商业模 式的创新。传统的以旧换新通常是一种促销手段，防 止消费者因耐用品还可再用而不买新产品。再制造以 旧换新, 则是顾客手中的旧产品对于生产企业来说具 有再利用的价值, 许多电子产品诸如手机均可回收再 利用。在当代社会, 气候变化、温室效应等问题逐渐 被全球所重视, 企业在生产过程中产生的碳排放亟待 管理, 在“构建人类命运共同体”提案中, 呼吁我们建 设一个清洁美丽的世界, 企业处于碳排放量较大的位 置理应做出应有贡献。

\section{1. 文献综述}

Saibal Ray,Tamer Boyaci,Necati Aras ${ }^{[1]}$ 等探究了市场细 分 (新老客户) 、所有顾客同价格以及价格歧视对以 旧换新耐用品制造商的定价影响;Raghunath Singh Rao,Om Narasimhan,George John ${ }^{[2]}$ 等分析了耐用消费品 市场的特点并以此向供应商提出建议。Kate J. Li,Duncan K. H. Fong,Susan H. Xu ${ }^{[3]}$ 等针对高科技企业 所面临的问题提出了在 $\mathrm{B} 2 \mathrm{~B}$ 市场上根据产品特点以及 客户的异质性提出了基于退货授权信息的客户细分和 产品退货预测方法; Jingna Ji,Zhiyong Zhang,Lei Yang ${ }^{[4]}$ 分析了一个集总量管制和交易管制以及消费者低碳偏 好于一体的详细模型, 发现当消费者的低碳敏感度满 足一定条件时，在线渠道的引入对厂商是有利的，当 消费者有强烈的低碳偏好时，供应链成员可以接受总 量管制和交易机制，并在此基础上比较了两种减排策 
2. 新产品与再制造产品实行价格歧视策略, 新产品价

略，包括生产策略中的单一制造商减排策略和联合减 排策略, 其中包括制造商和零售商的减排策略, 发现 联合减排策略对制造商和零售商都更有利; Rongyao $\mathrm{He}, \mathrm{Yu}$ Xiong,Zhibin Lin ${ }^{[5]}$ 评估了评估消费者搭便车对双 渠道闭环供应链产品生命周期碳排放的影响, 并评估 政府电子商务税对碳排放的影响, 研究表明尽管制造 商可以从消费者搭便车行为中获得经济利益, 但整个 供应链的碳排放总量也会增加, 政府对电子商务征税 有助于减少消费者搭便车和碳排放总量, 此外, 政府 可能不得不在最大限度地提高社会福利的前提下对电 子零售商进行补贴。由上所述, 多数研究研究集中于 碳规则条件下供应链各个节点的最佳策略、再制造决 策的工具、以旧换新 (回购) 的研究有耐用再制造产 品的最佳定价等, 关于 $\mathrm{O} 2 \mathrm{O}$ 供应链的研究相对较少, 本文在上述文章的基础上结合 $\mathrm{O} 2 \mathrm{O}$ 供应链的相关内容 加以探究。

\section{2. 模型变量设立与解释}

模型变量设立及解释由下表给出:

表 1 模型变量设置与解释

\begin{tabular}{|c|c|}
\hline 符号 & 含义 \\
\hline$p_{1}$ & 新产品零售价格 \\
\hline$p_{2}$ & 再制造产品零售价格 \\
\hline$p_{3}$ & $\begin{array}{l}\text { 以旧换新的补贴（零售商用以奖励旧顾客以 } \\
\text { 旧换新的补贴） }\end{array}$ \\
\hline$w_{1}$ & 新产品批发价格 \\
\hline$w_{2}$ & 再制造产品批发价格 \\
\hline$c_{1}$ & 新产品生产成本 \\
\hline$c_{2}$ & 再制造产品生产成本 \\
\hline$e_{1}$ & 制造单位新产品的碳排放 \\
\hline$e_{2}$ & 制造再制造产品的碳排放 \\
\hline$t$ & 政府制定的碳税率 \\
\hline$g$ & $\begin{array}{l}\mathrm{g} \text { 为常数, 对于制造商来说, 老顾客的旧产品 } \\
\text { 的平均剩余价值, 且 } \mathrm{g}<w_{2} \text {, 剩余价值不会 } \\
\text { 超过生产成本 }\end{array}$ \\
\hline$\theta$ & $\begin{array}{l}\text { 顾客对于新产品的购买意愿, } 0<\theta<1, \theta \\
\text { 在 }[0,1] \text { 上均匀分布 }\end{array}$ \\
\hline$\delta_{1}$ & 数值参数, $0<\delta_{1}<1$ \\
\hline$\delta_{2}$ & 数值参数, $0<\delta_{2}<\delta_{1}$ \\
\hline$Q_{\text {新产品 }}$ & 顾客对于新产品需求 \\
\hline$Q_{\text {再制造产品 }}$ & 顾客对于再制造产品需求 \\
\hline$Q_{\text {新顾客 }}$ & 新顾客对于所有产品的需求 \\
\hline$Q_{\text {老顾客 }}$ & 老顾客对于所有产品的需求 \\
\hline$a$ & 线上顾客比例, $0<a<1$ \\
\hline$b$ & 新顾客比例, $0<b<1$ \\
\hline
\end{tabular}

模型假设:

1. 零售商与制造商信息完全对称。零售商实行线上线 下同价策略, 出售两种产品, 新产品与再制造产品。 顾客购买时仅购买一个。
格高成本高, 生产单位新产品碳排放高, 即: $p_{1}>$ $p_{2}>p_{3}, c_{1}>c_{2}, e_{1}>e_{2}, w_{1}>w_{2}$ 。

3. 本文将顾客分为两种: 新顾客以及老顾客。新顾客 指不进行以旧换新的顾客, 老顾客指进行以旧换新购 买的顾客, 假设新顾客的比例为 $b$, 设在线上购买的比 例为 $a$ 。

4. 为使模型有意义, 假设 $p_{1}-p_{2}<1-\delta_{1}, p_{2}<$ $\delta_{1}, p_{1}-p_{3}<1-\delta_{2}, p_{2}-p_{3}<\delta_{1}-\delta_{2}$ 。

5.为了简化模型, 模型做归一化处理。

由于 $\mathrm{O} 2 \mathrm{O}$ 供应链线下与线下结合，信息通畅，打破了 渠道之间的壁垒, 因此顾客对于新产品的购买意愿均 为: $\theta$, 则顾客对于再制造产品的购买意愿: $\delta_{1} \theta$, $0<\delta_{1}<1$, 旧顾客对于其旧产品的评价: $\delta_{2} \theta$, $0<\delta_{2}<\delta_{1}$ 。由此新产品对于新顾客的效用为 $U_{1}=\theta-p_{1}$; 再制造产品对于新顾客的效用为 $U_{2}=\delta_{1} \theta-p_{2}$; 新产品对于老顾客的效用为 $U_{3}=$ $\theta-p_{1}+p_{3}$; 再制造产品对于老顾客的效用为 $U_{4}=\delta_{1} \theta-p_{2}+p_{3}$ 。

对线上新顾客: 当 $U_{1}>U_{2}$ 且 $U_{1}>0$ 时选择购买新 产品, 由 $U_{1}>U_{2} \Rightarrow \theta>\frac{p_{1}-p_{2}}{1-\delta_{1}}$, 由 $U_{1}>0 \Rightarrow$ $\theta>p_{1}$, 所以, 当同时满足 $\theta>\frac{p_{1}-p_{2}}{1-\delta_{1}}, \theta>p_{1}$ 时, 线上新顾客会购买新产品, 由于 $\theta$ 在 $[0,1]$ 上均匀分布, 需要判断 $p_{1}$ 与 $\frac{p_{1}-p_{2}}{1-\delta_{1}}$ 的大小关系 (为使模型有意义, 假 设 $p_{1}-p_{2}<1-\delta_{1}$, 即 $\frac{p_{1}-p_{2}}{1-\delta_{1}}<1$ )。假 设 $\frac{p_{1}-p_{2}}{1-\delta_{1}} \geq p_{1}$, 则 $p_{2} \leq \delta_{1} p_{1}$, 由此得出区间 $\left[\frac{p_{1}-p_{2}}{1-\delta_{1}}, 1\right]$ 为满足线上新顾客购买新产品的正概率区间, 因此 $p_{2} \leq \delta_{1} p_{1}$ 时线上新顾客对于新产品的需求 $Q_{1}=$ $a b\left(1-\frac{p_{1}-p_{2}}{1-\delta_{1}}\right)$; 若 $\frac{p_{1}-p_{2}}{1-\delta_{1}}<p_{1}$, 即 $p_{2}>\delta_{1} p_{1}$ 时, 满足条件的正概率区间为 $\left[p_{1}, 1\right]$, 此时线上新顾客 对于新产品的需求 $Q_{1}=a b\left(1-p_{1}\right)$ 。当购买新产 品的效用低于购买再制造产品, 即 $U_{1}<U_{2}$ 且 $U_{2}>$ 0 时, 购买再制造产品。由 $U_{1}<U_{2} \Rightarrow \theta<\frac{p_{1}-p_{2}}{1-\delta_{1}}$, 由 $U_{2}>0 \Rightarrow \theta>\frac{p_{2}}{\delta_{1}}$, 所以当同时满足 $\theta<\frac{p_{1}-p_{2}}{1-\delta_{1}}$, $\theta>\frac{p_{2}}{\delta_{1}}$ 时, 线上新顾客会购买再制造产品。判断 $\frac{p_{1}-p_{2}}{1-\delta_{1}}$ 与 $\frac{p_{2}}{\delta_{1}}$ 的大小关系。（假设 $p_{2}<\delta_{1}$, 即 $\frac{p_{2}}{\delta_{1}}<$ 1）假设 $\frac{p_{1}-p_{2}}{1-\delta_{1}} \geq \frac{p_{2}}{\delta_{1}}$, 则 $p_{2} \leq \delta_{1} p_{1}$, 此时满足条件 的 $\theta$ 的正概率区间为 $\left[\frac{p_{2}}{\delta_{1}}, \frac{p_{1}-p_{2}}{1-\delta_{1}}\right]$, 因此在 $p_{2} \leq$ $\delta_{1} p_{1}$ 的条件下线上新顾客对于再制造产品的需求 $Q_{2}=a b\left(\frac{p_{1}-p_{2}}{1-\delta_{1}}-\frac{p_{2}}{\delta_{1}}\right)$; 若 $\frac{p_{1}-p_{2}}{1-\delta_{1}}<\frac{p_{2}}{\delta_{1}}$, 即 $p_{2}>$ $\delta_{1} p_{1}$ 时, 同时满足上述的解集为空, 此时 $Q_{2}=0$ 。 
$b)\left(1-\frac{p_{1}-p_{3}}{1-\delta_{2}}\right)$, 购买再制造产品 $\theta$ 的正概率区间是 空集, 线上老顾客对于再制造产品的需求 $Q_{6}=0$ 。

4. 对线下老顾客, 当 $\Delta \geq 0$ 时, 线下老顾客对于新产 品的需求 $Q_{7}=(1-a)(1-b)\left(1-\frac{p_{1}-p_{2}}{1-\delta_{1}}\right)$, 对 于再制造产品的需求 $Q_{8}=(1-a)(1-$ b) $\left(\frac{p_{1}-p_{2}}{1-\delta_{1}}-\frac{p_{2}-p_{3}}{\delta_{1}-\delta_{2}}\right)$ 。当 $\Delta<0$ 时, 线下老顾客对于 新产品的需求 $Q_{7}=(1-a)(1-b)\left(1-\frac{p_{1}-p_{3}}{1-\delta_{2}}\right)$, 对于再制造产品的需求 $Q_{8}=0$ 。

易 知 : $Q_{\text {新产品 }}=Q_{1}+Q_{3}+Q_{5}+Q_{7}$;

$Q_{\text {再制造产品 }}=Q_{2}+Q_{4}+Q_{6}+Q_{8} ; Q_{\text {新顾客 }}=$ $Q_{1}+Q_{2}+Q_{3}+Q_{4} \quad ; \quad Q_{\text {老顾客 }}=Q_{5}+Q_{6}+$ $Q_{7}+Q_{8}$ 。因此, 需求函数可以汇总为下表

表 2 需求函数表

\begin{tabular}{|c|c|c|}
\hline & $\Delta \geq 0$ & $\Delta<0$ \\
\hline $\begin{array}{l}p_{2} \\
\leq \delta_{1} p_{1}\end{array}$ & $\begin{array}{l}Q_{\text {新产品 }}=1-\frac{p_{1}-p_{2}}{1-\delta_{1}} \\
Q_{\text {再制造产品 }}=\frac{p_{1}-p_{2}}{1-\delta_{1}}-b \frac{p_{2}}{\delta_{1}}-(1-b) \frac{p_{2}-p_{3}}{\delta_{1}-\delta_{2}} \\
Q_{\text {新顾客 }}=b\left(1-\frac{p_{2}}{\delta_{1}}\right) \\
Q_{\text {老顾客 }}=(1-b)\left(1-\frac{p_{2}-p_{3}}{\delta_{1}-\delta_{2}}\right)\end{array}$ & $\begin{array}{l}Q_{\text {新产品 }}=1-b \cdot \frac{p_{1}-p_{2}}{1-\delta_{1}}-(1-b) \frac{p_{1}-p_{3}}{1-\delta_{2}} \\
Q_{\text {再制造产品 }}=b \cdot\left(\frac{p_{1}-p_{2}}{1-\delta_{1}}-\frac{p_{2}}{\delta_{1}}\right) \\
Q_{\text {新顾客 }}=b\left(1-\frac{p_{2}}{\delta_{1}}\right) \\
Q_{\text {老顾客 }}=(1-b)\left(1-\frac{p_{1}-p_{3}}{1-\delta_{2}}\right)\end{array}$ \\
\hline $\begin{array}{l}p_{2} \\
>\delta_{1} p_{1}\end{array}$ & $\begin{array}{l}Q_{\text {新产品 }}=b\left(1-p_{1}\right)+(1-b)\left(1-\frac{p_{1}-p_{2}}{1-\delta_{1}}\right) \\
Q_{\text {再制造产品 }}=(1-b)\left(\frac{p_{1}-p_{2}}{1-\delta_{1}}-\frac{p_{2}-p_{3}}{\delta_{1}-\delta_{2}}\right) \\
Q_{\text {新顾客 }}=b\left(1-p_{1}\right) \\
Q_{\text {老顾客 }}=(1-b)\left(1-\frac{p_{2}-p_{3}}{\delta_{1}-\delta_{2}}\right)\end{array}$ & $\begin{array}{l}Q_{\text {新产品 }}=b\left(1-p_{1}\right)+(1-b)\left(1-\frac{p_{1}-p_{3}}{1-\delta_{2}}\right) \\
Q_{\text {再制造产品 }}=0 \\
Q_{\text {新顾客 }}=b\left(1-p_{1}\right) \\
Q_{\text {老顾客 }}=(1-b)\left(1-\frac{p_{1}-p_{3}}{1-\delta_{2}}\right)\end{array}$ \\
\hline
\end{tabular}

\section{3. 集中决策下O2O供应链最优产量与定价}

供应链的利润函数如下:

$\Pi_{\text {供应链 }}\left(p_{1}, p_{2}, p_{3}\right)=\left(p_{1}-c_{1}-e_{1} t\right) Q_{\text {新产品 }}+\left(p_{2}-c_{2}-\right.$ $\left.e_{2} t\right) Q_{\text {再制造产品 }}+\left(g-p_{3}\right) Q_{\text {老顾客 }}(3-1)$

1. 当 $c_{2}+e_{2} t \leq \delta_{1}\left(c_{1}+e_{1} t\right),\left(1-\delta_{1}\right) g \geq(1-$ $\left.\delta_{2}\right)\left(c_{2}+e_{2} t\right)-\left(\delta_{1}-\delta_{2}\right)\left(c_{1}+e_{1} t\right)$ 时, 供应链同时供 应新产品与再制造产品, 无论是在新顾客市场还是老 顾客市场, 最优定价 $p_{1}^{*}=\frac{1+c_{1}+e_{1} t}{2}, p_{2}^{*}=\frac{\delta_{1}+c_{2}+e_{2} t}{2}$, $p_{3}^{*}=\frac{\delta_{2}+g}{2}$; 最优产量 $Q_{\text {新产品 }}=\frac{1}{2}\left(1-\frac{c_{1}+e_{1} t-c_{2}-e_{2} t}{1-\delta_{1}}\right)$, $Q_{\text {再制造产品 }}=\frac{1}{2}\left(\frac{c_{1}+e_{1} t-c_{2}-e_{2} t}{1-\delta_{1}}-b \frac{c_{2}+e_{2} t}{\delta_{1}}-(1-\right.$ b) $\left.\frac{c_{2}+e_{2} t-g}{\delta_{1}-\delta_{2}}\right) 2$. 当 $c_{2}+e_{2} t>\delta_{1}\left(c_{1}+e_{1} t\right),\left(1-\delta_{1}\right) g \geq$ $\left(1-\delta_{2}\right)\left(c_{2}+e_{2} t\right)-\left(\delta_{1}-\delta_{2}\right)\left(c_{1}+e_{1} t\right)$ 时, 供应链只 在老顾客市场供应再制造产品, 此时新顾客不会购买 再制造产品; 当 $c_{2}+e_{2} t \leq \delta_{1}\left(c_{1}+e_{1} t\right),\left(1-\delta_{1}\right) g<$ $\left(1-\delta_{2}\right)\left(c_{2}+e_{2} t\right)-\left(\delta_{1}-\delta_{2}\right)\left(c_{1}+e_{1} t\right)$ 时，供应链只 在新顾客市场供应再制造产品, 此时老顾客不会购买 再制造产品; 以上两种市场时, 最优定价 $p_{1}^{*}=\frac{1+c_{1}+e_{1} t}{2}$, $p_{2}^{*}=\frac{\delta_{1}+c_{2}+e_{2} t}{2}, p_{3}^{*}=\frac{\delta_{2}+g}{2}$; 最优产量分别为 $Q_{\text {新产品 }}=$ $\frac{b}{2}\left(1-c_{1}-e_{1} t\right)+\frac{1-b}{2}(1-$

$\left.\frac{c_{1}+e_{1} t-c_{2}-e_{2} t}{1-\delta_{1}}\right), Q_{\text {再制造产品 }}=\frac{1-b}{2}\left(\frac{c_{1}+e_{1} t-c_{2}-e_{2} t}{1-\delta_{1}}-\right.$ $\left.\frac{c_{2}+e_{2} t-g}{\delta_{1}-\delta_{2}}\right) ; Q_{\text {新产品 }}=\frac{1}{2}\left(1-b \frac{c_{1}+e_{1} t-c_{2}-e_{2} t}{1-\delta_{1}}-(1-\right.$

b) $\left.\frac{c_{1}+e_{1} t-g}{1-\delta_{2}}\right), Q_{\text {再制造产品 }}=\frac{b}{2}\left(\frac{c_{1}+e_{1} t-c_{2}-e_{2} t}{1-\delta_{1}}-\frac{c_{2}+e_{2} t}{\delta_{1}}\right)$ 。 
3. 当 $c_{2}+e_{2} t>\delta_{1}\left(c_{1}+e_{1} t\right),\left(1-\delta_{1}\right) g<(1-$ $\left.\delta_{2}\right)\left(c_{2}+e_{2} t\right)-\left(\delta_{1}-\delta_{2}\right)\left(c_{1}+e_{1} t\right)$ 时, 供应链停止供 应再制造产品, 无论是在新顾客市场还是老顾客市场, 此时最优定价 $p_{1}^{*}=\frac{1+c_{1}+e_{1} t}{2}, p_{3}^{*}=\frac{\delta_{2}+g}{2}$; 最优产量 $Q_{\text {新产品 }}=\frac{b}{2}\left(1-c_{1}-e_{1} t\right)+\frac{1-b}{2}\left(1-\frac{c_{1}+e_{1} t-g}{1-\delta_{2}}\right)$, $Q_{\text {再制造产品 }}=0$ 。

证明: 当 $p_{2} \leq \delta_{1} p_{1}, \Delta \geq 0$ 时, $Q_{\text {新产品 }}=1-\frac{p_{1}-p_{2}}{1-\delta_{1}}$, $Q_{\text {再制造产品 }}=\frac{p_{1}-p_{2}}{1-\delta_{1}}-b \frac{p_{2}}{\delta_{1}}-(1-b) \frac{p_{2}-p_{3}}{\delta_{1}-\delta_{2}} \quad, \quad Q_{\text {老顾客 }}=$ $(1-b)\left(1-\frac{p_{2}-p_{3}}{\delta_{1}-\delta_{2}}\right)$, 将 $Q_{\text {新产品 }} 、 Q_{\text {再制造产品 }} Q_{\text {老顾客 }}$ 代入 式 (3-1)

$\Pi_{\text {供应链 }}\left(p_{1}, p_{2}, p_{3}\right)$ 分别 对 $p_{1}, p_{2}, p_{3}$ 求偏导且令 $\frac{\partial \Pi_{\text {供应链 }}\left(p_{1}, p_{2}, p_{3}\right)}{\partial p_{1}}=0 \quad, \quad \frac{\partial \Pi_{\text {供应链 }}\left(p_{1}, p_{2}, p_{3}\right)}{\partial p_{2}}=0 \quad$, $\frac{\partial \Pi_{\text {供应链 }}\left(p_{1}, p_{2}, p_{3}\right)}{\partial p_{3}}=0$; 联立求解可得

$p_{1}^{*}=\frac{1+c_{1}+e_{1} t}{2}, p_{2}^{*}=\frac{\delta_{1}+c_{2}+e_{2} t}{2}, p_{3}^{*}=\frac{\delta_{2}+g}{2}$

将 $p_{1}^{*}, p_{2}^{*}, p_{3}^{*}$ 代入 $p_{2} \leq \delta_{1} p_{1}, \Delta \geq 0$ 中可得 $c_{2}+e_{2} t \leq$ $\delta_{1}\left(c_{1}+e_{1} t\right), \quad\left(1-\delta_{1}\right) g \geq\left(1-\delta_{2}\right)\left(c_{2}+e_{2} t\right)-\left(\delta_{1}-\right.$ $\left.\delta_{2}\right)\left(c_{1}+e_{1} t\right)$, 将最有定价带入需求函数中即可得 1 。 对于 $2 、 3$, 原理相同, 不再赘述。

由 $\frac{\partial p_{1}}{\partial t}=\frac{e_{1}}{2}>0, \frac{\partial p_{2}}{\partial t}=\frac{e_{2}}{2}>0, \frac{\partial p_{3}}{\partial t}=0$ 且 $\frac{\partial p_{1}}{\partial t}=\frac{e_{1}}{2}>$ $\frac{\partial p_{2}}{\partial t}=\frac{e_{2}}{2}$, 可知新产品价格与再制造产品价格随着碳税 率的提高而提高, 并且新产品价格提高的速率比再制 造产品要快。

由最优产量知, 当再制造产品生产成本变小而新产品 生产成本不变或增大时, 市场对于再制造产品的需求 量会增加, 而当再制造产品生产成本变大而新产品生 产成本不变或变小时, 市场对于再制造产品的需求量 会减小, 直到再制造产品的需求量为 0 . 对于管理者而 言, 在其他条件不变的情况下, 零售价格只与生产成 本与碳税率相关, 因此需要时刻关注生产成本与碳税 率的关系。在生产成本与碳税率变化时, 要相应的增 加或减少再制造与新产品的价格以及改变再制造产品 的供应情况。

\section{4.分散决策下 $\mathrm{O} 2 \mathrm{O}$ 供应链最优产量与定价}

零售商、制造商利润表达式:

$\Pi_{\text {零售商 }}\left(p_{1}, p_{2}, p_{3}\right)=\left(p_{1}-w_{1}\right) Q_{\text {新产品 }}+$

$\left(p_{2}-w_{2}\right) Q_{\text {再制造产品 }}-p_{3} Q_{\text {老顾客 }}$ (4-1)

$\Pi_{\text {制造商 }}\left(w_{1}, w_{2}\right)=\left(w_{1}-c_{1}-e_{1} t\right) Q_{\text {新产品 }}+\left(w_{2}-c_{2}-\right.$ $\left.e_{2} t\right) Q_{\text {再制造产品 }}+g Q_{\text {老顾客 }}(4-2)$

1. 当 $p_{2} \leq \delta_{1} p_{1}, \Delta \geq 0$ 时, $Q_{\text {新产品 }}=1-\frac{p_{1}-p_{2}}{1-\delta_{1}}$, $Q_{\text {再制造产品 }}=\frac{p_{1}-p_{2}}{1-\delta_{1}}-b \frac{p_{2}}{\delta_{1}}-(1-b) \frac{p_{2}-p_{3}}{\delta_{1}-\delta_{2}}, \quad Q_{\text {老顾客 }}=$ $(1-b)\left(1-\frac{p_{2}-p_{3}}{\delta_{1}-\delta_{2}}\right)$, 代入 (4-1) 中分别对 $p_{1}, p_{2}, p_{3}$ 求偏导并 令 $\frac{\partial \Pi_{\text {零售商 }}\left(p_{1}, p_{2}, p_{3}\right)}{\partial p_{1}}=0, \frac{\partial \Pi_{\text {零售商 }}\left(p_{1}, p_{2}, p_{3}\right)}{\partial p_{2}}=0$, $\frac{\partial \Pi_{\text {零售商 }}\left(p_{1}, p_{2}, p_{3}\right)}{\partial p_{3}}=0$; 联立求解可得 $p_{1}^{*}=\frac{1+w_{1}}{2}, p_{2}^{*}=$ $\frac{\delta_{1}+w_{2}}{2}, p_{3}^{*}=\frac{\delta_{2}}{2}$, 此时零售商会获得最大利润, 但此时 $w_{1}, w_{2}$ 未知, 将由制造商利润函数求解。将求解出的 $p_{1}^{*}, p_{2}^{*}, p_{3}^{*}$ 代入需求函数得 $Q_{\text {新产品 }}=\frac{1}{2}\left(1-\frac{w_{1}-w_{2}}{1-\delta_{1}}\right)$, $Q_{\text {再制造产品 }}=\frac{1}{2}\left(\frac{w_{1}-w_{2}}{1-\delta_{1}}-b \frac{w_{2}}{\delta_{1}}-(1-b) \frac{w_{2}}{\delta_{1}-\delta_{2}}\right), Q_{\text {老顾客 }}=$ $\frac{1}{2}(1-b)\left(1-\frac{w_{2}}{\delta_{1}-\delta_{2}}\right)$ 。再将上述需求函数代入 (4-2) 得 分别对 $w_{1}, w_{2}$ 求偏导并令 $\frac{\partial \Pi_{\text {制造商 }}\left(w_{1}, w_{2}\right)}{\partial w_{1}}=0$, $\frac{\partial \Pi_{\text {制造商 }}\left(w_{1}, w_{2}\right)}{\partial w_{2}}=0$; 联立求解可得 $w_{1}^{*}=\frac{1}{2}\left(1+c_{1}+e_{1} t-\right.$ $\left.\frac{(1-b) \delta_{1}\left(g+\delta_{2}\right)}{\delta_{1}-b \delta_{2}}\right), \quad w_{2}^{*}=\frac{1}{2}\left(c_{2}+e_{2} t+\frac{\delta_{1}\left(\delta_{1}-\delta_{2}\right)-(1-b) \delta_{1} g}{\delta_{1}-b \delta_{2}}\right)$, 此时制造商获得最大利润, 将上述 $w_{1}^{*}, w_{2}^{*}$ 代入 $p_{1}^{*}, p_{2}^{*}$, $p_{3}^{*}$ 可得: $p_{1}^{*}=\frac{1}{2}+\frac{1}{4}\left(1+c_{1}+e_{1} t-\frac{(1-b) \delta_{1}\left(g+\delta_{2}\right)}{\delta_{1}-b \delta_{2}}\right), p_{2}^{*}=$ $\frac{\delta_{1}}{2}+\frac{1}{4}\left(c_{2}+e_{2} t+\frac{\delta_{1}\left(\delta_{1}-\delta_{2}\right)-(1-b) \delta_{1} g}{\delta_{1}-b \delta_{2}}\right), p_{3}^{*}=\frac{\delta_{2}}{2} ;$ 最优产 量 $\quad Q_{\text {新产品 }}=1-\frac{1}{1-\delta_{1}}\left[\frac{1}{2}+\frac{1}{4}\left(1+c_{1}+e_{1} t-\right.\right.$ $\left.\frac{(1-b) \delta_{1}\left(g+\delta_{2}\right)}{\delta_{1}-b \delta_{2}}\right)-\frac{\delta_{1}}{2}-\frac{1}{4}\left(c_{2}+e_{2} t+\right.$ $\left.\left.\frac{\delta_{1}\left(\delta_{1}-\delta_{2}\right)-(1-b) \delta_{1} g}{\delta_{1}-b \delta_{2}}\right)\right], Q_{\text {再制造产品 }}=\frac{1}{1-\delta_{1}}\left[\frac{1}{2}+\frac{1}{4}(1+\right.$ $\left.c_{1}+e_{1} t-\frac{(1-b) \delta_{1}\left(g+\delta_{2}\right)}{\delta_{1}-b \delta_{2}}\right)-\frac{\delta_{1}}{2}-\frac{1}{4}\left(c_{2}+e_{2} t+\right.$ $\left.\left.\frac{\delta_{1}\left(\delta_{1}-\delta_{2}\right)-(1-b) \delta_{1} g}{\delta_{1}-b \delta_{2}}\right)\right]-\frac{b}{\delta_{1}}\left[\frac{\delta_{1}}{2}+\frac{1}{4}\left(c_{2}+e_{2} t+\right.\right.$ $\left.\left.\frac{\delta_{1}\left(\delta_{1}-\delta_{2}\right)-(1-b) \delta_{1} g}{\delta_{1}-b \delta_{2}}\right)\right]-\frac{1-b}{\delta_{1}-\delta_{2}}\left[\frac{\delta_{1}}{2}+\frac{1}{4}\left(c_{2}+e_{2} t+\right.\right.$ $\left.\left.\frac{\delta_{1}\left(\delta_{1}-\delta_{2}\right)-(1-b) \delta_{1} g}{\delta_{1}-b \delta_{2}}\right)-\frac{\delta_{2}}{2}\right]$ 。

2. 当 $p_{2} \leq \delta_{1} p_{1}, \Delta<0$ 时, $Q_{\text {新产品 }}=1-b \cdot \frac{p_{1}-p_{2}}{1-\delta_{1}}-$ $(1-b) \frac{p_{1}-p_{3}}{1-\delta_{2}}, \quad Q_{\text {再制造产品 }}=b \cdot\left(\frac{p_{1}-p_{2}}{1-\delta_{1}}-\frac{p_{2}}{\delta_{1}}\right), \quad Q_{\text {老顾客 }}=$ $(1-b)\left(1-\frac{p_{1}-p_{3}}{1-\delta_{2}}\right)$, 计算方法与上述相同, 此时 $w_{1}^{*}=$ $\frac{1}{2}\left(c_{1}+e_{1} t+\frac{1-\delta_{2}-(1-b) g}{1-b \delta_{2}}\right) \quad, \quad w_{2}^{*}=\frac{1}{2}\left(c_{2}+e_{2} t+\right.$ $\left.\frac{\delta_{1}\left(1-\delta_{2}\right)-(1-b) \delta_{1} g}{1-b \delta_{2}}\right), \quad p_{1}^{*}=\frac{1}{2}+\frac{1}{4}\left(c_{1}+e_{1} t+\frac{1-\delta_{2}-(1-b) g}{1-b \delta_{2}}\right)$, $p_{2}^{*}=\frac{\delta_{1}}{2}+\frac{1}{4}\left(c_{2}+e_{2} t+\frac{\delta_{1}\left(1-\delta_{2}\right)-(1-b) \delta_{1} g}{1-b \delta_{2}}\right), \quad p_{3}^{*}=\frac{\delta_{2}}{2} ; \quad$ 最 优 产 量 $Q_{\text {新产品 }}=1-\frac{b}{1-\delta_{1}}\left[\frac{1}{2}+\frac{1}{4}\left(c_{1}+e_{1} t+\right.\right.$ $\left.\frac{1-\delta_{2}-(1-b) g}{1-b \delta_{2}}\right)-\frac{\delta_{1}}{2}-\frac{1}{4}\left(c_{2}+e_{2} t+\right.$ $\left.\left.\frac{\delta_{1}\left(1-\delta_{2}\right)-(1-b) \delta_{1} g}{1-b \delta_{2}}\right)\right]-\frac{1-b}{1-\delta_{2}}\left[\frac{1}{2}+\frac{1}{4}\left(c_{1}+e_{1} t+\right.\right.$ $\left.\left.\frac{1-\delta_{2}-(1-b) g}{1-b \delta_{2}}\right)-\frac{\delta_{2}}{2}\right], Q_{\text {再制造产品 }}=\frac{b}{1-\delta_{1}}\left[\frac{1}{2}+\frac{1}{4}\left(c_{1}+\right.\right.$ $\left.e_{1} t+\frac{1-\delta_{2}-(1-b) g}{1-b \delta_{2}}\right)-\frac{\delta_{1}}{2}-\frac{1}{4}\left(c_{2}+e_{2} t+\right.$ $\left.\left.\frac{\delta_{1}\left(1-\delta_{2}\right)-(1-b) \delta_{1} g}{1-b \delta_{2}}\right)\right]-\frac{b}{\delta_{1}}\left[\frac{\delta_{1}}{2}+\frac{1}{4}\left(c_{2}+e_{2} t+\right.\right.$ $\left.\left.\frac{\delta_{1}\left(1-\delta_{2}\right)-(1-b) \delta_{1} g}{1-b \delta_{2}}\right)\right]$ 。

3. 当 $p_{2}>\delta_{1} p_{1}, \Delta \geq 0$ 时, $Q_{\text {新产品 }}=b\left(1-p_{1}\right)+(1-$ $b)\left(1-\frac{p_{1}-p_{2}}{1-\delta_{1}}\right) ， Q_{\text {再制造产品 }}=(1-b)\left(\frac{p_{1}-p_{2}}{1-\delta_{1}}-\frac{p_{2}-p_{3}}{\delta_{1}-\delta_{2}}\right)$, $Q_{\text {老顾客 }}=(1-b)\left(1-\frac{p_{2}-p_{3}}{\delta_{1}-\delta_{2}}\right)$, 此 时 $w_{1}^{*}=\frac{1}{2}\left(c_{1}+e_{1} t+\right.$ $\left.\frac{1-\delta_{2}-(1-b) g}{1-b \delta_{2}}\right), \quad w_{2}^{*}=\frac{1}{2}\left(c_{2}+e_{2} t+\frac{\delta_{1}-\delta_{2}-\left(1-b \delta_{1}\right) g}{1-b \delta_{2}}\right), \quad p_{1}^{*}=$ $\frac{1}{2}+\frac{1}{4}\left(c_{1}+e_{1} t+\frac{1-\delta_{2}-(1-b) g}{1-b \delta_{2}}\right), \quad p_{2}^{*}=\frac{\delta_{1}}{2}+\frac{1}{4}\left(c_{2}+e_{2} t+\right.$ 
$p_{2}^{*}=\frac{\delta_{1}}{2}+\frac{1}{4}\left(c_{2}+e_{2} t+\frac{\delta_{1}\left(\delta_{1}-\delta_{2}\right)-(1-b) \delta_{1} g}{\delta_{1}-b \delta_{2}}\right), p_{3}^{*}=\frac{\delta_{2}}{2}$ 。零

$\left.\frac{\delta_{1}-\delta_{2}-\left(1-b \delta_{1}\right) g}{1-b \delta_{2}}\right), p_{3}^{*}=\frac{\delta_{2}}{2}$; 最优产量 $Q_{\text {新产品 }}=b(1-$ $\left.\frac{1}{2}-\frac{1}{4}\left(c_{1}+e_{1} t+\frac{1-\delta_{2}-(1-b) g}{1-b \delta_{2}}\right)\right)+(1-b)\{1-$ $\frac{1}{1-\delta_{1}}\left[\frac{1}{2}+\frac{1}{4}\left(c_{1}+e_{1} t+\frac{1-\delta_{2}-(1-b) g}{1-b \delta_{2}}\right)-\frac{\delta_{1}}{2}-\frac{1}{4}\left(c_{2}+\right.\right.$ $\left.\left.\left.e_{2} t+\frac{\delta_{1}-\delta_{2}-\left(1-b \delta_{1}\right) g}{1-b \delta_{2}}\right)\right]\right\}, Q_{\text {再制造产品 }}=(1-$ b) $\left\{\frac{1}{1-\delta_{1}}\left[\frac{1}{2}+\frac{1}{4}\left(c_{1}+e_{1} t+\frac{1-\delta_{2}-(1-b) g}{1-b \delta_{2}}\right)-\frac{\delta_{1}}{2}-\right.\right.$ $\left.\frac{1}{4}\left(c_{2}+e_{2} t+\frac{\delta_{1}-\delta_{2}-\left(1-b \delta_{1}\right) g}{1-b \delta_{2}}\right)\right]-\frac{1}{\delta_{1}-\delta_{2}}\left[\frac{\delta_{1}}{2}+\frac{1}{4}\left(c_{2}+\right.\right.$ $\left.\left.\left.e_{2} t+\frac{\delta_{1}-\delta_{2}-\left(1-b \delta_{1}\right) g}{1-b \delta_{2}}\right)-\frac{\delta_{2}}{2}\right]\right\}$ 。

4. 当 $p_{2}>\delta_{1} p_{1}, \Delta<0$ 时, $Q_{\text {新产品 }}=b\left(1-p_{1}\right)+(1-$ b) $\left(1-\frac{p_{1}-p_{3}}{1-\delta_{2}}\right)=1-b p_{1}-(1-b) \frac{p_{1}-p_{3}}{1-\delta_{2}}, Q_{\text {再制造产品 }}=$ $0, Q_{\text {老顾客 }}=(1-b)\left(1-\frac{p_{1}-p_{3}}{1-\delta_{2}}\right)$, 此 时 $w_{1}^{*}=\frac{1}{2}\left(c_{1}+\right.$ $\left.e_{1} t+\frac{1-\delta_{2}-(1-b) g}{1-b \delta_{2}}\right), \quad w_{2}^{*}=0, \quad p_{1}^{*}=\frac{1}{2}+\frac{1}{4}\left(c_{1}+e_{1} t+\right.$ $\left.\frac{1-\delta_{2}-(1-b) g}{1-b \delta_{2}}\right), p_{2}^{*}=0, p_{3}^{*}=\frac{\delta_{2}}{2}$; 最 优产 量 $Q_{\text {新产品 }}=$ $b\left(1-\frac{1}{2}-\frac{1}{4}\left(c_{1}+e_{1} t+\frac{1-\delta_{2}-(1-b) g}{1-b \delta_{2}}\right)\right)+(1-$ b) $\left\{1-\frac{1}{1-\delta_{2}}\left[\frac{1}{2}+\frac{1}{4}\left(c_{1}+e_{1} t+\frac{1-\delta_{2}-(1-b) g}{1-b \delta_{2}}\right)-\right.\right.$ $\left.\left.\frac{\delta_{2}}{2}\right]\right\}, Q_{\text {再制造产品 }}=0$ 。

综合可得, 在不同情况下所得到的最优零售定价相同, 即 $p_{1}^{*}=\frac{1+w_{1}}{2}, p_{2}^{*}=\frac{\delta_{1}+w_{2}}{2}, p_{3}^{*}=\frac{\delta_{2}}{2}$, 生产价格越高, 零 售价格越高, 无论是新产品还是再制造产品, 以旧换 新价格补贴为常数, 只与老顾客对于其手中旧产品的 评价参数 $\delta_{2}$ 有关, 老顾客对于其手中的旧产品评价越 高, 以旧换新的价格补贴就越高, 与常识相符。不同 情形下共有四种最优生产策略, 当 $\Delta \geq 0, p_{2} \leq \delta_{1} p_{1}$ 时, 即 $w_{2} \leq \delta_{1} w_{1} ，\left(\delta_{1}-\delta_{2}\right) w_{1} \geq\left(1-\delta_{2}\right) w_{2}$ 时， $w_{1}^{*}=$ $\frac{1}{2}\left(1+c_{1}+e_{1} t-\frac{(1-b) \delta_{1}\left(g+\delta_{2}\right)}{\delta_{1}-b \delta_{2}}\right) \quad, \quad w_{2}^{*}=\frac{1}{2}\left(c_{2}+e_{2} t+\right.$ $\left.\frac{\delta_{1}\left(\delta_{1}-\delta_{2}\right)-(1-b) \delta_{1} g}{\delta_{1}-b \delta_{2}}\right)$, 新产品与再制造产品在新顾客市场 与老顾客市场均有供应。观察可知, 两种产品的批发 价格均与生产成本、碳排放、碳税率以及剩余价值常 数 $\mathrm{g}$ 有关。由 $\frac{\partial w_{1}^{*}}{\partial c_{1}}=\frac{1}{2}=\frac{\partial w_{2}^{*}}{\partial c_{2}}>0$, 新产品和再制造产品 批发价格保持恒定速率随着对应生产成本的提高而提 高, 并且速率相同, 均为 $\frac{1}{2}$ 。由 $\frac{\partial w_{1}^{*}}{\partial e_{1}}=\frac{t}{2}=\frac{\partial w_{2}^{*}}{\partial e_{2}} \geq 0$, 两 种产品批发价格随碳排放变化的速率相同, 均为 $\frac{t}{2}$, 碳 税率越高, 在其他条件不变的情况下, 批发价格随碳 排放提高而提高的速率越高, 相对的, 随碳排放降低 而降低的速率也越高, 因此政府可通过改变碳税率来 调节企业生产与碳排放; 由 $\frac{\partial w_{1}^{*}}{\partial t}=\frac{e_{1}}{2}>\frac{\partial w_{2}^{*}}{\partial t}=\frac{e_{2}}{2}$, 可知 生产价格随着碳税率的提高而提高, 并且新产品对碳 税率的变化更敏感, 这是因为新产品的碳排放更高。 当制造商作出决策后, 零售商根据生产价格进行最终 决策。此时 $p_{1}^{*}=\frac{1}{2}+\frac{1}{4}\left(1+c_{1}+e_{1} t-\frac{(1-b) \delta_{1}\left(g+\delta_{2}\right)}{\delta_{1}-b \delta_{2}}\right)$,
售价格随着生产成本的增加而增加, 并且是恒定速率 $\frac{1}{4}$, 新产品与再制造产品速率相等 $\left(\frac{\partial p_{1}^{*}}{\partial c_{1}}=\frac{1}{4}=\frac{\partial p_{2}^{*}}{\partial c_{2}}\right)$ 。零售 价格随着碳税率的增加而增加, 并且新产品的增加速 率大于再制造产品的增加速率 $\left(\frac{\partial p_{1}^{*}}{\partial t}=\frac{e_{1}}{4}>\frac{\partial p_{2}^{*}}{\partial t}=\frac{e_{2}}{4}\right)$ 。 与供应链集中决策以及制造商相比, 零售商受到生产 成本以及碳税率的影响要更弱一些, 这是因为供应链 集中决策时, 将零售商以及制造商看做一个整体, 所 以对制造商的影响直接反映到了零售价格上; 分散决 策时, 生产成本以及碳税率是直接是施加到制造商的 身上, 而经过了供应链内部的博弯后使得生产价格以 及碳税率对于零售价格的影响得到了削弱。

\section{5. 结论}

本文建立了集中于分散决策下的最优定价与生产策略, 最优产量决策与生产成本之间的关系以及消费者对于 再制造产品以及旧产品的评价有关，最优定价决策与 生产成本以及碳税率有关, 不同情况下进行不同的定 价。当外界因素例如人力成本、原材料价格等影响到 生产成本时, 零售商可以及时调整零售策略, 制造商 可以追随零售商调整生产策略; 当生产成本、碳税率 综合动态变化时, 零售商可以调整零售价格并将市场 需求反馈给制造商, 制造商由此追随零售商调整批发 价格并调整产量。对于政府, 可以通过调整碳税率来 迫使供应链作出应对已达到其促进经济发展、保护环 境的目的, 例如通过提高碳税率来刺激行业内节能减 排技术的发展，通过政府渠道宣传再制造以旧换新的 绿色节能意义来促进更多消费者购买再制造产品来帮 助生产企业缓解碳税率所带来的成本压力。

\section{REFERENCES}

[1] Saibal Ray,Tamer Boyaci,Necati Aras. Optimal Prices and Trade-in Rebates for Durable, Remanufacturable Products[J]. Manufacturing \& Service Operations Management,2005,7(3).

[2]Raghunath Singh Rao,Om Narasimhan,George John. Understanding the Role of Trade-Ins in Durable Goods Markets: Theory and Evidence[J]. Marketing Science,2009,28(5).

[3] Kate J. Li,Duncan K. H. Fong,Susan H. Xu. Managing Trade-in Programs Based on Product Characteristics and Customer Heterogeneity in Business-to-Business Markets[J]. Manufacturing \& Service Operations Management,2011,13(1).

[4] Jingna Ji,Zhiyong Zhang,Lei Yang. Carbon emission reduction decisions in the retail-/dual-channel supply 
chain with consumers' preference[J]. Journal of Cleaner Production, 2017,141.

[5] Rongyao He, Yu Xiong,Zhibin Lin. Carbon emissions in a dual channel closed loop supply chain: the impact of consumer free riding behavior[J]. Journal of Cleaner Production,2016,134.

[6] Aussadavut Dumrongsiri,Ming Fan,Apurva Jain,Kamran Moinzadeh. A supply chain model with direct and retail channels[J]. European Journal of Operational Research,2006,187(3).

[7]Urvashi,S.R. Singh,N. Singh. Green Supply Chain Model with Product Remanufacturing under Volume Flexible Environment[J]. Procedia Technology,2013,10.

[8]Chen, K., Kaya, M., \& Özer, Ö.Dual sales channel management with service competition.Manufacturing \& Service Operations Management; Linthicum Vol. 10, Iss. 4, (Fall 2008): 654-675.

[9]Janice E. Carrillo,Asoo J. Vakharia,Ruoxuan Wang. Environmental implications for online retailing[J]. European Journal of Operational Research,2014,239(3).

[10]Shaofu Du,Wenzhi Tang,Malin Song. Low-carbon production with low-carbon premium in cap-and-trade regulation[J]. Journal of Cleaner Production,2016,134.

[11]Ma Zu-Jun,Nian Zhang,Ying Dai,Shu Hu. Managing channel profits of different cooperative models in closed-loop supply chains[J]. Omega,2016,59.

[12]Zhi Pei,Ruiliang Yan. National advertising, dualchannel coordination and firm performance[J]. Journal of Retailing and Consumer Services,2013,20(2).

[13]Juhong Gao,Hongshuai Han,Liting Hou,Haiyan Wang. Pricing and effort decisions in a closed-loop supply chain under different channel power structures[J]. Journal of Cleaner Production,2016,112. 